\title{
Research on the Development Trend of Intelligent Vehicle
}

\author{
Xiumin Ye \\ Centre for Informatization Study of Chinese Academy of Social Sciences, Beijing, China
}

\begin{abstract}
This article has given the intelligent vehicle concept and analyzed six development trends, including market growth accelerated, core technology intelligentized, connection networked, resource integration platform-based, cross-border cooperation ecologicalized and business model shared. We should grasp the rules of the development of the intelligent vehicle market, which is helpful for the companies to seize the opportunity to meet the challenges of the era of intelligent vehicles.
\end{abstract}

\section{Keywords-intelligent vehicle; artificial intelligence; big data}

\section{INTRODUCTION}

With the industrial innovation driven by the development of the Internet, communication technology, cloud computing and artificial intelligence technology, together with the innovation of clean energy alternative to fossil fuel energy, auto industry is facing a major change. The intelligent vehicle era is coming. The inherent system and pattern of the automotive industry will be broken under the big changes. The risks and opportunities will be coexisting. The intelligent vehicle market will become the focus of competition. On the one hand, the traditional automobile enterprises are also sensitive to the opportunity and challenge in the changing period. Almost all of the traditional automobile enterprises are driving in independent development, and most of them have set commercial automatic driving goal in advance to 2020. On the other hand, due to the significant reduction in the threshold of automobile manufacturing, a number of high-tech giant company and start-up companies have cross-border entered for the intelligent vehicle market. This paper focuses on the analysis of the development trend of the intelligent vehicle. Its aim is to cause a wider range of discussion about the industry, so that the law of development of intelligent vehicle industry will be more and clearer to guide the practice effectively.

\section{THE CONCEPT OF INTELLIGENT VeHICLES}

There are a number of concepts about intelligent vehicle. The main three are: intelligent vehicle, intelligent network vehicles and unmanned vehicle. There are subtle differences among the three concepts, which we should differentiate clearly.

Intelligent vehicle is a comprehensive system. It integrates the functions of environmental perception, planning and decision making, and multi grade driving. It is based on the technology of modern computer, sensing, information fusion, communication, artificial intelligence and automatic control. Intelligent vehicle is a typical high-tech synthesis, including intelligent driving system, life service system, security protection system, location service system and service system.

Compared with concept of intelligent vehicle, intelligent networked vehicle place more emphasis on the extent of the networked. It also always appears in the government documents. The definition of intelligent networked vehicle, which is come from Automotive Industry Alliance, is a new generation of vehicles with complex environment perception, intelligent decision-making, coordination control and execution function. In order to realize these functions, it must be equipped with advanced on-board sensors and other devices. Moreover, it must integrate modern communication and network technology in order to exchange the information among people, vehicles and roads, etc. The key word "networked" in the concept of intelligent networked vehicle means to realize the information exchange among the passenger, the car, the cloud and the environment.

The primary stage of the development of intelligent vehicles is the application of driving assistance system and automatic driving system. The most advanced stage is driverless vehicles. Driverless vehicles, also known as the automatic driving vehicles, can know the road environment with the sensing system. It also can plan the best route automatically and control of vehicle to reach the destination smoothly.

\section{DEVELOPMENT TREND OF THE INTELLIGENT VEHICLE}

\section{A. Intelligent Vehicle is about to Usher in a Period of Rapid Growth}

After decades of exploration and accumulation, the intelligent vehicle industry is about to enter a period of rapid growth. In recent years, Investment by the giant enterprises in the field of intelligent vehicle is increasing continuously. The continuous technological innovation is very active. Some of the intelligent module has been put into practical application. Along with the original solid base of car production and the continued good policy, intelligent vehicles will usher in a rapid development period.

The rapid development of intelligent vehicles is mainly in the following five aspects: first, accelerate innovation, including intelligence and related technologies, business models, business management, business ecosystem construction, etc... Second, it is about to enter the stage of mass production from the stage of development. Third, degree of intelligence of intelligent vehicles is increasing gradually, from the wide use of intelligent module until the driverless vehicles available. Fourth, the Internet and the automotive field are 
penetrating mutually and integrating rapidly. More and more giant enterprises are participating in the construction and application of intelligent vehicles. Fifth, there are a series of good news: the supporting environment is constantly optimized; People's understanding of intelligent vehicles is further and deeply; the industrial capital is inflowing steadily; infrastructure continues to improve, of favorable policies are introduced frequently.

\section{B. More Intelligent, until Completely Unmanned}

The intelligent vehicle is improving the level of intelligence gradually, until it will realize automation completely. Intelligent vehicle covers four kinds of ability. That is: perception, thinking, action and learning. This capacity will continue to improve with the pace of technological innovation. Moreover, this capacity will also integrate with each other perfectly until to fully realize intelligent.

Perception is the ability to get real-time traffic scenes and other environmental information and to convert them to the standard data timely. The application of the Internet of things, sensors and computing technology provides the basis for the realization of the sensing ability. Sensing components, like the human body's ear, nose and throat organs, can feel, recognize and receive feedback from the road, pedestrians, other vehicles, weather and other environments. This information includes not only digital information, but also sound information, three-dimensional image information. In addition, the operation status of the car itself, wear parts is also recorded in real time. The information received is stored into standard format data in the temporary database according to a certain standard. Then the data is fed back to the cloud analysis system quickly.

Thinking ability is the ability to filter, analyze, judge and make decisions. Based on the modern information technology, such as cloud computing and data mining, intelligent vehicle can classify and analyze the sensed data. As in the path planning, it can distinguish all of the routes ahead. It can calculate the congestion status and time that would take on each path. It can analyze the passenger's demand. It can decide which path should be chosen. On the basis of analysis and comparison, it can choose a set of accurate, efficient and feasible driving scheme in the alternative scheme.

Action is the ability to accurately execute the decisions, communicate, operate, control and service. When the vehicle receives the action instruction, the instruction information is transmitted to each system. Division and cooperation of all parts ensure that the action instructions will be completed successfully. Such as driving to the designated location along the best route automatically, automatic parking advanced waiting for passengers at the designated location, etc...

Learning ability is the key to improve the level of intelligence. In the process of continuously interacting with the environment and the passengers, the intelligent vehicle continuously accumulates and stores valuable knowledge and data. The knowledge is continuously improved, iterated, upgraded and accumulated as experience. For example, the intelligent vehicle can perceive the passenger's characteristics and provide the corresponding services based on the past experience. When there are children in the passengers, the intelligent vehicle can determine the age according to the child's height and weight, and then it can provide the appropriate air conditioning temperature, and recommend the appropriate age of cartoons or songs. These are all based on the past experience that it has learnt.

The most advanced development stage of intelligent vehicle is unmanned. In the unmanned state, the intelligent vehicle on the one hand to realize full automatic driving. It can deliver passengers to the destination safely and efficiently without the driver. Under the control of adults, even a child can also take a safe ride alone by a driverless car. On the other hand, according to passenger information, unmanned vehicles can provide a comfortable environment, adjust the seat height and the temperature of the air conditioning, plan the best route, recommended matching entertainment etc... Finally, the $l$ the driverless car will liberate the driver and release lot of space. People can make full use of the vehicle space to complete a variety of things, such as video conference, writing speeches, doing homework, online purchase of daily necessities, and even sleeping comfortably.

\section{Interconnection of all Things to Enhance the Level of Intelligent}

Networking to keep the information free flow is the premise and basis for the realization of intelligent. Networking is one of the most important trends of intelligent vehicle. The Internet is the transmission carrier of information. It can also be called an intelligent vehicle nervous system. It organically connects people, cars and the environment. The networking of intelligent vehicles includes the interconnection of people and vehicles, the car and the car, the vehicle and the cloud, the vehicle and the road, the car and the environment. Information flows by the Internet to make the intelligent vehicle realize the whole process of perception, analysis, decision-making and control. The accuracy, timeliness, smoothness and security of information flow play a key role in the normal operation of intelligent vehicles.

The extent of the networking determines the degree of intelligent. Only by the networking, can the real-time information obtaining and sharing be realized. Only by the networking, can the data perceived be sent to control systems and cloud in time. Only by networking, can the big data analysis and cloud computing results be feedback to the car timely. Only by the network, can the thousands of parts work harmoniously in division and cooperation. Only by the network, can people enjoy a variety of services in the automotive space, such as video business talks, online shopping, remote control home appliances.

\section{Platform Based}

The intelligent vehicle will perceive abundant and complicated information while running. The information has to be processed into data of the standard format. After storage and transmission, the data can be analyzed and dealt. One of the most important symbols that the upgrade from the traditional car to the intelligent vehicle is the height data-based. All the information, such as vehicle information, driver information, environmental information, traffic information, service information, will highly achieve data-based. The intelligent vehicle relies on big data to support decision making. 
With the development of cloud computing and big data technology, it is possible to analyze and mine the massive data. Intelligent vehicle in operation will real-time access to a large number of data. The data can be analyzed and calculated in demand and the way easy to expand through the cloud platform. Intelligent vehicles interact with the cloud in real time to obtain the required data and conclusions, for example, the best road to choose, the passenger' s need to identify, etc..

In the future, intelligent vehicle is an open platform to accommodate a variety of hardware and software, applications and services. Intelligent vehicles like the current smart phones will include a variety of applications. Users can download a variety of APP based on individual preferences. For example, video services will come from major film companies, ordering service will include all kinds of restaurants.

\section{E. The Ecology of Cross Border Cooperation}

Intelligent vehicle is not only a machine of transport, which is also equipped with a variety of software, applications and services. Therefore, the intelligent vehicle can be seen as a platform linking between supply and demand. Platform coupled with the system environment constitutes a small ecological, which improves continuously as a business ecosystem. The system also includes a rich body with hierarchy. The demand body is transportation users; the supply body is a variety of services and applications such as video providers, content providers, e-commerce sellers and so on. Service providers include auto manufacturers, cloud computing providers, providers, telecommunications and other accessories. System environment included infrastructure construction, relevant laws and regulations, technological innovation, etc.

With the increasing complexity of the intelligent vehicle business ecosystem, any enterprise can't do all the business due to the resources limited. Social division and collaboration has become the most effective way for production. Social collaboration pays more emphasis on resource sharing and complementary advantages, so as to achieve the purpose of win-win. Division and cooperation can not only give full play to the professional expertise of each subject, but also to meet the diverse needs of all kinds of users. The division and cooperation includes not only the internal division, but also the social division and cooperation among enterprises. The cooperation among enterprises and partners is entirely in accordance with the need to establish a cooperative relationship, which is the process of self-organization.

In the process of the evolution of the intelligent vehicle ecosystem, the main body cooperates with each other and interacts with the environment. At last, it evolves into an interdependent ecosystem. Intelligent vehicle is involved in many segments of the industry. Many areas have exceeded the automobile industry boundaries. All kinds of complementary must take complementary advantages and cross-border cooperation, so that it can be organized into an organic interaction ecosystem to achieve a win-win situation together.

Cross border cooperation has become a major feature of the research and development phase of intelligent vehicle in the world. Shanghai Automobile Group will join together with Alibaba to develop Internet automotive ecosystem; Beijing
Automotive Group and Le Group will hold together to create intelligent vehicle; Foxconn Technology Group and Tencent have carried out the cooperation on internet+ intelligent electric vehicle. In the field of subdivision, capital cooperation has become the shortcut to start the market. Asia Pacific shares a motor company; Junsheng Electronics Ltd. Has bought equity from Car Voice Ltd., etc...

The more the main types of services provided and the more closer the connection on the intelligent vehicle platform, the more stable and healthy will the ecosystem be. Otherwise, the imbalance of the development of the main body or the fault of the relationship, will lead to the platform system into a low level of disorderly development, even into collapse in serious condition. In the future, the competition among enterprises will be transformed into the competition between the value chain and ecosystem.

\section{F. Business Model Sharing}

In the future, along with the development of networking and intelligence, car sharing will subvert the existing sales model. The auto market will realize "Lease instead of purchase”, "on-demand travel services”, "personalized service” and "data-driven services". This model innovation will promote the transformation of traditional manufacturing to services.

First of all, "Lease instead of purchase" can rent a car at any time needed and pay on demand. The consumers can enjoy a variety of personalized services. Consumers can rent a suitable idle car by the internet platform to achieve car sharing without buying a car. The consumers can rent every kinds of can in accordance with the characteristics of personalized travel. When the family is going to travel, you can rent a caravan or SUV by the platform; you can rent high-end commercial vehicles if you take part in a business talk. You can rent a truck if you want to move furniture...... In the era of unmanned driving, the elderly and children can also call a car to enjoy the specialized service. It is available for the family member to control the main parameters of the can by the mobile phone. Sharing mode allows the travel to become freer, more environmentally friendly, more convenient, more efficient, more economical and lower carbon. In the original travel mode, a family has at least one car, which has exacerbated the road congestion, parking space shortage, environmental pollution and the waste of resources. Sharing mode can reduce the purchase cost and maintenance cost. Moreover, one needn't rent a special parking space. At the same time, one can make use of car rental services to meet the needs.

Secondly, the platform can improve service quality using big data technology to allocate the resources scientifically. According to the user's data, the platform analyzes the demand characteristics of each region. Then the platform can select the location of the parking lot and assign the car resources according the analysis result. The platform can select the relatively idle land mass to construct the parking lot, which will be managed intelligently. It is good to release a large number of urban areas and road space. It is also helpful to ease urban congestion. Urban space saved can be used to build more commercial and service facilities to facilitate the needs of 
residents. The platform can also provide a complete set of travel solutions for the consumer, together with car rental plans, travel plans, tourist spots and hotel recommendations.

Finally, the sharing business model will also promote the global revenue significantly. According to McKinsey predicts that the sharing service model will contribute additional revenue of about 1 trillion and 500 billion U.S. dollars by 2030, while the traditional automotive industry revenue is approximately $\$ 5$ trillion and 200 billion U.S. dollars, compared to $\$ 3$ trillion and 500 billion in 2015, an increase of up to $50 \%$. Over the past five years, the average growth rate of global auto sales remained at the level of 3.6\%. Automotive market has been saturated in the United States and Japan and other major automobile producing countries. The sale of the passenger car has lead to even negative growth.

The sharing business model has brought new business opportunities in the context of the global gloomy automotive market. It is reported that Volkswagen, BMW, Mercedes Benz are trying to explore the sharing of business model. They will change the model from cars sale into cars rental. Toyota Corporation is very optimistic about the sharing business model. It believes that the number of vehicles to provide sharing service will reach ten million by 2020. TOYOTA even hopes to involve in the field of auto finance with sharing model.

\section{- Case Study}

\section{Soda Technology: Car Sharing Mode}

The aim of Soda Technology is to create a new way of travel. It takes new energy intelligent vehicle as an entry point, applying big data and mobile Internet technology, together combining with sharing model. Opening Soda Travel APP, the users can simply find the shared car nearby, and the users can complete the whole renting procedure by the phone, including a virtual key lock, a key to start the vehicle, mobile payments, etc... Although the user has not bought a car and parking spaces, but he still can travel conveniently.

\section{Preparing Actively to Embrace the Change}

Intelligence is leading the automotive industry a major change. Intelligent vehicles will become another important new entrance of the Internet. It will be the commanding heights of the industrial chain. Intelligent vehicle will have a profound impact on the economic and social development. It will enhance traffic travel experience and promote the transformation of traditional automotive industry.

Change is coming, the government, enterprises and consumers should grasp the development trend of the intelligent vehicle market, be ready for this intelligent revolution from the thought and the action. We should seize the opportunity and accumulate competitive advantage to meet the intelligent vehicle era.

\section{REFERENCES}

[1] Talal AI-Shihabi, Ronald R.Mourant, "Toward More Realistic Driving Behavior Models for Automatic Vehicles in Driving Simulators", the Transportation Research Board's 82nd Annual Meeting, 2003.

[2] Th. Fraichard, Ph. Gamier, "Fuzzy Control to drive car like vehicles", Robotics and Autonomous Systems, 34(2001)1 - 22.
[3] Thomas Hessburg, Masayoshi Tomizuka,"Fuzzy Logic Control for Lane Change Maneuvers in Lateral Vehicle Guidance", California PATH Working Paper, UCB-ITS-PWP-95-13, Oct 1995.

[4] Thomas Mc Avoy, "Intelligent'Control' Applications in the Process Industries", Annual Reviews in Control 26(2002) 75-86.

[5] Malcolm J.A. Strens, and Ian N. Gregory, Tracking in cluttered images, Image and Vision Computing, 2003, 21(1), 891 - 911.

[6] Peihua Li, Tianwen Zhang, and Arthur E. C. Pece, Visual contour tracking based on particle filters, Image and Vision Computing, 2003, 21(1), 111-123.

[7] Tygn-Luh Liu, and Hwann-Tzong Chen, Real-time tracking using trust-region methods, IEEE Trans-actions on Pattern Analysis and Machine Intelligence, 2004, 26(3), 397-402.

[8] Michael Chapman. Using Vehicle Probe Data to Diagnose Road Weather Conditions-Results from the Detroit IntelliDrive SM [C ] M Washington, D.C, State Transportation Departments, TRB2010 Annual Meeting.2010:1-16.

[9] Tao W, Zi-you G, Xiao-mei Z. Multiple velocity difference model and its stability analysis[J].2006:634-640.

[10] Wei, J., Dolan, J. M.,\& Litkouhi, B. (2013). Autonomous vehicle social behavior for highway entrance ramp management. IEEE intelligent vehicles symposium, Gold Coast, QLD, Queensland, Australia (pp. 201-207). 